\title{
SonHi-C: a set of non-procedural approaches for predicting 3D genome organization from Hi-C data
}

\author{
Kimberly MacKay ${ }^{1}$, Mats Carlsson ${ }^{2}$ and Anthony Kusalik ${ }^{1}$ \\ ${ }^{1}$ Department of Computer Science, University of Saskatchewan, Saskatoon, SK, Canada \\ ${ }^{2}$ RISE SICS, Kista, Sweden
}

\section{Abstract}

Background: Many computational methods have been developed that leverage the results from biological experiments (such as Hi-C) to infer the 3D organization of the genome. Formally, this is referred to as the $3 \mathrm{D}$ genome reconstruction problem (3D-GRP). None of the existing methods for solving the 3D-GRP have utilized a non-procedural programming approach (such as constraint programming or integer programming) despite the established advantages and successful applications of such approaches for predicting the 3D structure of other biomolecules. Our objective was to develop a set of mathematical models and corresponding non-procedural implementations for solving the 3D-GRP to realize the same advantages.

Results: We present a set of non-procedural approaches for predicting 3D genome organization from Hi-C data (collectively referred to as SonHi-C and pronounced "sonic"). Specifically, this set is comprised of three mathematical models based on constraint programming (CP), graph matching (GM) and integer programming (IP). All of the mathematical models were implemented using non-procedural languages and tested with Hi-C data from Schizosaccharomyces pombe (fission yeast). The CP implementation could not optimally solve the problem posed by the fission yeast data after several days of execution time. The 
GM and IP implementations were able to predict a 3D model of the fission yeast genome in 1.088 and 294.44 seconds, respectively. These 3D models were then biologically validated through literature search which verified that the predictions were able to recapitulate key documented features of the yeast genome.

Conclusions: Overall, the mathematical models and programs developed here demonstrate the power of non-procedural programming and graph theoretic techniques for quickly and accurately modelling the 3D genome from Hi-C data. Additionally, they highlight the practical differences observed when differing non-procedural approaches are utilized to solve the 3D-GRP.

Key Words: 3D Genome Reconstruction Problem, Mathematical Modelling, Constraint Programming, Graph Matching, Integer Programming

\section{Background}

Within the nucleus, a cell's genetic information undergoes extensive folding and reorganization throughout normal physiological processes. Just like in origami where the same piece of paper folded in different ways allows the paper to take on different forms and potential functions, it is possible that different genomic organizations are related to various nuclear functions. Until recently, it has been extremely difficult to comprehensively investigate this relationship due to the lack of high-resolution and high-throughput techniques for identifying genomic organizations. The development of a technique called Hi-C (based on chromosome conformation capture) [36] has made it possible to detect the complete set of genomic regions in close physical proximity. This proximity is often referred to as an "interaction" between two genomic regions. These interactions can be categorized as either intra-chromosomal (cis) interactions or inter-chromosomal (trans) interactions (Figure 1).

It is currently unknown whether the $3 \mathrm{D}$ genomic organization drives various nuclear functions or vice versa. Alterations in the $3 \mathrm{D}$ organization of chromosome territories have been 


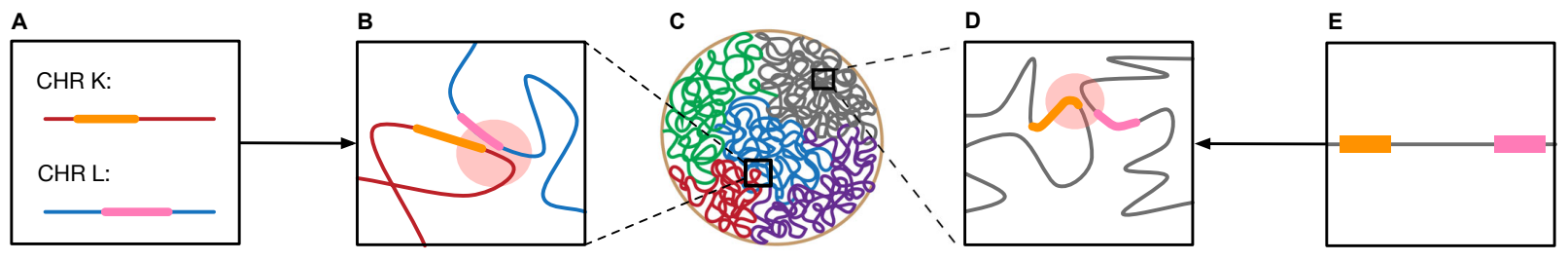

Figure 1: A representation of the DNA-DNA interactions that can occur within the 3D genome structure. Panels give the following representations. A: the linear locations of the genes undergoing a trans-interaction between two hypothetical chromosomes, K and L. B: a trans-interaction. C: a nucleus with the coloured lines representing the separate chromosomes from Babaei et al. [3]. D: a cis-interaction. These genes might be linearly "distant" but still have a detectable interaction in 3D space. E: the linear locations of the genes that are undergoing a 3D cis-interaction. The orange and pink regions in panels A, B, D and E are examples of possible gene locations. The red circles in panels $\mathrm{B}$ and $\mathrm{D}$ represent the genomic regions involved in an interaction.

demonstrated in a wide variety of cellular processes, including differentiation [31], serum response [39], therapeutic response [21, 40] and response to DNA damage [41]. The unique spatial organization of the genome that is seen under these different cellular conditions is hypothesized to be a crucial mechanism driving various nuclear and cellular functions. It has been theorized that this dynamic organization of the genome may be driven by the global regulation of gene expression [1, 7, 11]. Therefore, the identification of distinct genome interactions may highlight novel mechanisms responsible for organism health and development.

$\mathrm{Hi}-\mathrm{C}$ [36] is a biological technique that utilizes next generation sequencing technologies to detect regions of the genome that are interacting in 3D space. These regions may be located on different chromosomes or distally on the same chromosome. An overview of the experimental procedure is depicted in Figure 2. Briefly, (1) cells are fixed with formaldehyde in order to covalently cross-link genomic regions that are in close 3D proximity. (2) The crosslinked fragments are then digested with a restriction enzyme to remove the potentially large non-interacting interconnecting segments of DNA. (3) The sticky ends generated through the restriction digest in step (2) are filled in with biotinylated nucleotides. (4) Digested fragments are ligated together. (5) The initial cross-linking is removed, resulting in DNA fragments that 
represent the two genomic regions that form an interaction. (6) The biotinylated products are purified using streptavidin beads allowing for the detection of fragments that were cut by restriction enzymes. (7) Paired-end sequencing is then performed and the resultant reads are mapped to a reference genome using a Hi-C specific read mapper [2].

Mapping the raw data of a Hi-C experiment to a reference genome results in the generation of a $N \times N$ matrix (a whole-genome contact map) where $N$ is the number of "bins" which represent linear regions of genomic DNA. In general, the number of genomic bins is approximately equal to the total genome size divided by the Hi-C experimental resolution. Whole-genome contact maps are characteristically sparse and symmetric along the diagonal. Each cell $\left(A_{i, j}\right)$ of a hypothetical whole-genome contact map (A) records the count of how many times the genomic bin $i$ was found to interact with the genomic bin $j$. These counts are often referred to as the frequency of the interaction between $A_{i}$ and $A_{j}$ (or interaction frequency). Inherent systematic biases within the whole-genome contact map are dampened by normalizing the interaction frequencies. Typically, an iterative correction and eigenvector decomposition (ICE) [24] or Knight-Ruiz (KR) [27, 35] normalization are/is applied to the raw data resulting in fractional interaction frequencies.

These normalized whole-genome contact maps can be used to infer the 3D organization of the genome. The process of predicting a model of the 3D genomic organization from a contact map is known as the 3D genome reconstruction problem (3D-GRP) [50]. Typically this is done by converting the normalized interaction frequencies into a set of corresponding pairwise Euclidean distances. In general it is assumed that a pair of genomic regions with a higher interaction frequency will often be closer in 3D space than a pair of genomic regions with a lower interaction frequency [14, 23, 34]. Most computational tools for solving the 3D-GRP then take the predicted pairwise Euclidean distances and produce a visualization of the 3D genome by modelling the chromatin fibre as a polymer [52]. In general, most existing programs can be broadly classified as either (1) consensus or (2) ensemble methods. Consensus methods generate a single population-averaged genomic model that best 


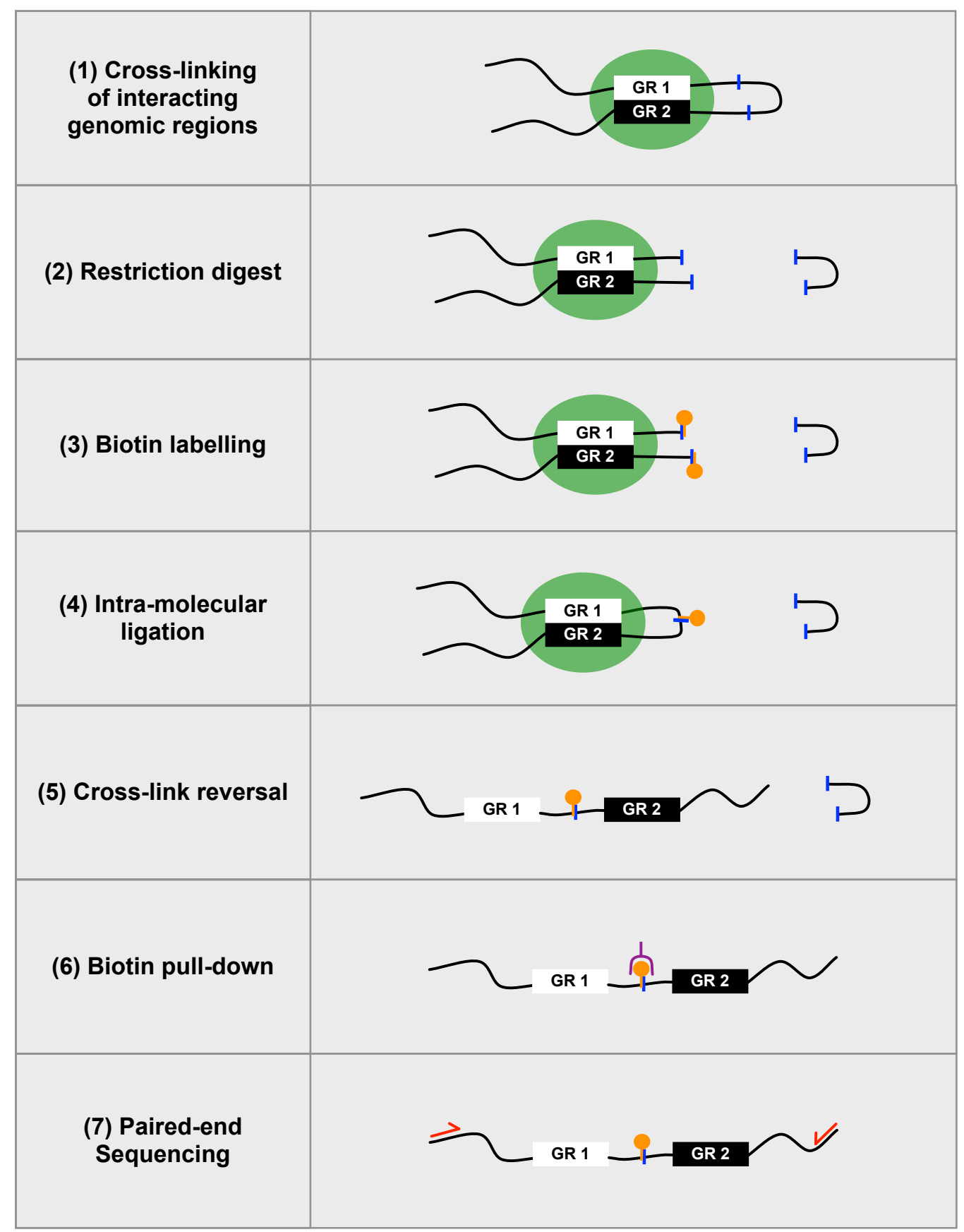

Figure 2: A simplified overview of the Hi-C protocol adapted from reference [36]. GR stands for "genomic region". The blue lines represent the location of a restriction enzyme cut site; green circles, a pair of genomic regions being chemically cross-linked together; orange circles, biotin; and red arrows, the primers that are required for paired-end sequencing. The purple symbol represents a streptavidin bead that can be used to purify molecules with a biotin label. 
represents the whole-genome contact map, while ensemble methods produce a collection of genome models that represent the inherent heterogeneity of genome organizations within a population of cells [33].

None of the current methods to solve the 3D-GRP have used a non-procedural approach (such as constraint programming (CP), integer programming (IP) or mixed-integer programming (MIP)) even though non-procedural approaches have been successfully used to predict the 3D structure of other biomolecules [5, 20, 29, 30, 45, 46, 47, 59. These applications are advantageous since they have been shown to produce more biologically relevant results and take less computational time when compared to competing methods [16, 17, 46]. One of the advantages of utilizing non-procedural programming in biological applications is that biological knowledge can be naturally encoded into the program, instead of having to convert this information into procedural steps. Additionally, they restrict the search space of possible solutions and ensure only models that agree with constraint-encoded experimental data are retained [26]. It is expected that similar advantages will be achieved by applying non-procedural programming to predicting the 3D genomic organization. Here we present three mathematical modelling solutions to the 3D-GRP, collectively referred to as sonHiC (pronounced "sonic"). Each mathematical model was implemented in a non-procedural language and tested with a normalized whole-genome contact map from fission yeast (Gene Expression Omnibus accession number: GSM1379427) [42]. Fission yeast was selected since it is a well-studied model organism with a relatively small but complex genome [58]. Additionally, it has many properties governing genomic organization that have been previously established using a variety of microscopy techniques.

\section{Results \& Discussion}

This section presents the results for three mathematical modelling solutions to the 3D-GRP (Subsection 3.1), the non-procedural implementation for each mathematical model (Subsec- 
tion 3.2 and a visualization of the predicted genome model (Subsection 3.3). Subsection

3.4 demonstrates the effect of varying the $m$ parameter (described below) for the fission yeast dataset and Subsection 3.5 discusses how this research could be applied to organisms with higher ploidies (the number of chromosome copies) and/or larger genomes. All of the auxiliary files and programs used to generate these results are available at the project home page (https://github.com/kimmackay/SonHi-C).

\subsection{Mathematical Modelling}

Under normal cellular conditions, a given genomic region can be simultaneously involved in more than one interaction within the genome [18]. In contrast, a single genomic region within an individual cell is only able to participate in one $\mathrm{Hi}-\mathrm{C}$ mediated interaction due to inherent restrictions within the biochemistry of the $\mathrm{Hi}-\mathrm{C}$ experimental protocol [56]. In diploid organisms (organisms with two genomic copies) single cell Hi-C reactions are only able to detect two Hi-C mediated interactions per genomic region, one for each genomic copy [43]. An analogous restriction can be assumed in haploid organisms (organisms with only one genomic copy), where a single genomic region can only be actively detected in one Hi-C mediated interaction in a single cell. Using this restriction, a model of the $3 \mathrm{D}$ genome organization can be constructed from a whole-genome contact map by selecting a ploidydependent subset of the interactions for each genomic region that maximizes the sum of the corresponding interaction frequencies. The mathematical models and programs presented in this paper focus on modelling the 3D organization of haploid genomes but, as outlined in Subsection 3.5, they could be easily extended to organisms with higher ploidies.

Naively, a greedy heuristic could be employed to model the 3D fission yeast genome organization using the strategy described above. Briefly, the subset of interactions representing the solution set would be chosen by sorting and selecting the interactions with the largest corresponding frequency values. This process would then be repeated, rejecting any frequency that involves a region of the genome that has already been selected. This heuristic will fail 
to take into account the situation where lower frequencies, which were rejected by selecting a higher frequency interaction, actually result in a greater overall maximum value for the sum of all selected frequencies within the solution. An example of this can be seen in Figure 3 where panel $\mathrm{A}$ is a hypothetical whole-genome contact map and panels $\mathrm{B}$ and $\mathrm{C}$ represent two possible solution matrices with different overall frequency sums. Specifically, Figure 3B follows the greedy heuristic described above which results in a non-optimal solution where the selected frequencies sum to 1.3. Figure $3 \mathrm{C}$ shows the optimal solution where the selected frequencies sum to 1.4. This type of optimization problem has been shown to be well-suited for non-procedural approaches.

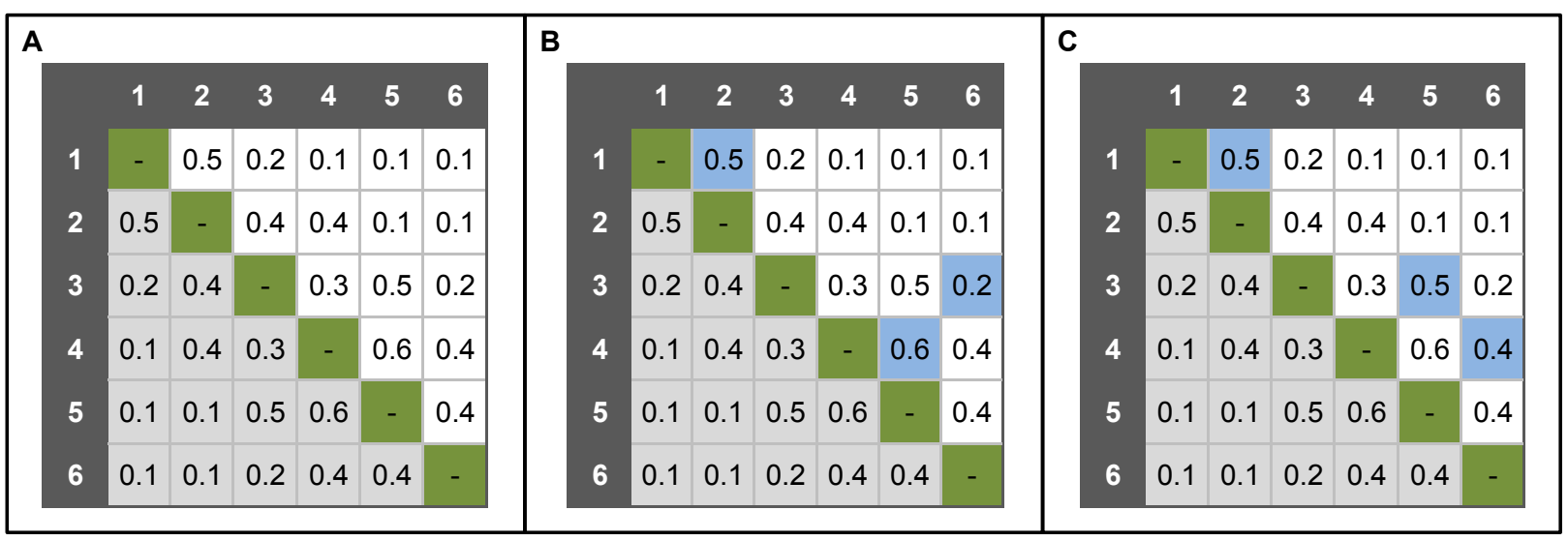

Figure 3: An example of two (of many) possible solutions to a 3D genome reconstruction problem. For all of the panels: the symmetric lower half of the contact map is indicated in light grey, the diagonal that represents "self-self" interactions is indicated in green and the genomic bin labels are represented in dark grey. For panels B and C: the blue boxes represent the subset of frequencies that could be selected as possible solutions (for $m=1$ ). Panel $\mathrm{B}$ is a representation of a valid, non-optimal solution from the greedy algorithm and panel $\mathrm{C}$ is a representation of the valid optimal solution for the contact map where the sum of the selected interaction frequencies are 1.3 and 1.4 , respectively.

We developed three mathematical solutions to the 3D-GRP which describe the relationships present within the whole-genome contact map. As mentioned previously, a wholegenome contact map is a $N \times N$ matrix where the genome has been partitioned into $N$ genomic bins. For a hypothetical whole-genome contact map $(\mathbf{A})$, each cell $\left(A_{i, j}\right)$ records the normalized interaction frequency between genomic bins $i$ and $j$. By construction, the 
contact map is symmetric $\left(A_{i, j}=A_{j, i}\right.$ for all $\left.i, j\right)$, and its main diagonal elements are all zero $\left(A_{i, i}=0\right.$ for all $\left.i\right)$. The second parameter of our mathematical models is the maximal number of interactions that a given genomic bin can be involved in based on the source organism's ploidy (denoted by the parameter $m$ ). For instance, $m$ would be set to the following values based on the number of chromosome copies present: $m=1$ (haploid), $m=2$ (diploid; common in mammals), $m=4$ (tetraploid; common in plants), and so on. Below are the specifics for three mathematical formulations of the 3D-GRP based on a given whole-genome contact map (A). Some variants require that the interaction frequencies be rounded and scaled to integer values.

\subsubsection{Constraint Programming (CP)}

Our first model, CP, is encoded with Constraint Programming [49]. This model is valid for $m=1$ only and requires integral $A_{i, j}$ values due to the implementation (described in Subsection 3.2.2). It is based on introducing variables $M_{i}$ where $M_{i}=j$ if genomic bin $i$ interacts with genomic bin $j$, and $M_{i}=i$ otherwise. The goal of this model is to solve $M_{i}$ for all $i$. The model is given in Mathematical Model 1 . Since this model encodes a combinatorial problem, its time complexity is exponential in the worst case.

maximize

$$
\sum_{i \in V} A_{i, M_{i}}
$$

subject to:

$$
\begin{gathered}
M_{i}=j \leftrightarrow M_{j}=i, \forall i, j \in V \\
M_{i} \in\{i\} \cup\left\{j \mid A_{i, j}>0\right\}, \forall i \in V
\end{gathered}
$$

Mathematical Model 1: The CP model, valid for $m=1$ and integral interaction frequencies $\left(A_{i, j}\right)$ only. $V$ is the set $\{1, \ldots, N\}$ representing the genomic bins. 


\subsubsection{Graph Matching (GM)}

Our second model, GM, is only valid for the $m=1$ case. By representing the contact map as an undirected graph with $N$ vertices (genomic bins) and $\frac{N(N-1)}{2}$ edges (interactions) the 3D-GRP can be regarded as the problem of computing a maximum-weight matching for the graph $G=(V, E)$. A matching in a graph is a set of edges where no two edges share an endpoint. Each edge has an associated weight, and the weight of the matching is simply the sum of the weights of the edges in the matching. In the GM model, the vertices $V$ are the set of genomic bins, the edges $E$ are the set $\left\{(i, j) \mid i<j \wedge A_{i, j}>0\right\}$, and the weights are given by $A$. An $O(|V| \cdot|E| \log |V|)$ implementation of the weighted matching problem was reported by Mehlhorn and Schäfer [38], and is provided in the LEDA algorithm library ${ }^{1}$. However, this formulation does not guarantee that each vertex in the original graph is represented in the matching. In terms of the 3D-GRP, this means that there is no guarantee each genomic bin from the contact map would be represented in the solution. In order to overcome this, the 3D-GRP can be represented as a maximum-weight perfect matching problem to ensure all vertices in the graph are matched. Edmonds [15] invented the first polynomial maximum-weight perfect matching algorithm, which runs in $O\left(|V|^{2}|E|\right)$ time. If the graph was completely connected (i.e. $|E|=\frac{N(N-1)}{2}$ ), this would intuitively suggest a time complexity of $O\left(N^{4}\right)$, but in reality whole-genome contact maps are characteristically sparse resulting in $|E| \ll N^{2}$ since zero-weight edges are not represented in the graph. As such, the mean computational complexity will depend on the experimental resolution and resultant sparsity of a given whole-genome contact map. Kolmogorov's Blossom V algorithm [28] is considered the most efficient implementation of Edmonds algorithm. We use this reduction in our model, given in Mathematical Model 2.

\footnotetext{
1 http://www .algorithmic-solutions.com/
} 
Solve the maximum-weight perfect matching problem for the graph $G^{\prime}=\left(V^{\prime}, E^{\prime}\right)$ and weight function $f: E^{\prime} \mapsto \mathbb{R}$, i.e.:

maximize

$$
\sum_{(i, j) \in E^{\prime \prime}} f(i, j)
$$

subject to:

$$
\begin{gathered}
V^{\prime}=\{i \mid i \in V\} \cup\{i+N \mid i \in V\} \\
E^{\prime}=\{(i, j) \mid(i, j) \in E\} \cup\{(i+N, j+N) \mid(i, j) \in E\} \cup\{(i, i+N) \mid i \in V\} \\
E^{\prime \prime} \subseteq E^{\prime} \text { is a perfect matching for } G^{\prime} \\
f(i, j)= \begin{cases}A_{i, j} & \text {, if } i \leq N \wedge j \leq N \\
A_{i-N, j-N} & , \text { if } i>N \wedge j>N \\
0 & , \text { otherwise }\end{cases}
\end{gathered}
$$

Mathematical Model 2: The GM model, for $m=1$ only. $V$ is the set $\{1, \ldots, N\}$ representing the genomic bins. $E$ is the set $\left\{(i, j) \mid i<j \wedge A_{i, j}>0\right\}$ representing the interactions and the weights are given by $A . f(i, j)$ is the function used to calculate edge weight. $G^{\prime}=\left(V^{\prime}, E^{\prime}\right)$ is an extended graph used to map $G=(V, E)$ to a maximumweight perfect matching problem. This mapping to maximum-weight perfect matching was given by Mehlhorn [38, footnote 1].

\subsubsection{Integer Programming (IP)}

Our third model, IP, uses Integer Programming [57] and is valid for any value of $m$. It is based on introducing variables $x_{i, j}$ that assume a value of 1 if genomic bin $i$ interacts with genomic bin $j$, and 0 otherwise. The goal of this model is to solve $x_{i, j}$ for all $i, j$. The model is given in Mathematical Model 3. Similarly to the CP model, this model encodes a combinatorial problem resulting in an exponential worst-case time complexity. 
maximize

$$
\sum_{(i, j) \in E} A_{i, j} x_{i, j}
$$

subject to:

$$
\begin{gathered}
\sum_{(i, j) \in E} x_{i, j}+\sum_{(j, i) \in E} x_{j, i} \leq m, \forall i \in V \\
x_{i, j} \in\{0,1\}, \forall(i, j) \in E
\end{gathered}
$$

Mathematical Model 3: The IP model, for any $m$. $V$ is the set $\{1, \ldots, N\}$ representing the genomic bins. $E$ is the set $\left\{(i, j) \mid i<j \wedge A_{i, j}>0\right\}$ representing the interactions and the weights are given by $A$.

\subsection{Implementations}

Each mathematical model described above was implemented in a non-procedural language (specifics are provided below) and tested with an existing fission yeast Hi-C dataset (GEO accession number: GSM1379427 [42]). These implementations were run on a server-grade computer with sufficient main memory to represent the entire problem. When the implementations were run on the complete fission yeast whole-genome contact map (results presented in Subsections 3.2.3 and 3.2.4, there were only a few trans-chromosomal interactions within the solution sets making it difficult to infer the organization of the chromosomes in relation to each other. The low number of trans-chromosomal interactions is likely due to the fact that cis-chromosomal interactions are known to have higher interaction frequencies than trans-chromosomal interactions within the genome [12, 33]. This makes it more likely for cis-chromosomal interactions to be included in the solution set since the goal of the mathematical models described above is to select a maximal subset of interaction frequencies. To overcome this, a divide and conquer approach was used where each cis-chromosomal and pairwise trans-chromosomal subproblem was locally solved. These solutions were then merged to retain the selected cis- and trans-chromosomal interactions from each subproblem (described in Subsection 3.2.1). Overall, this divide and conquer approach resulted in a 
larger number of trans-chromosomal interactions being included in the final solution set.

\subsubsection{Divide \& Conquer}

As mentioned above, the cis-chromosomal subproblems better represent the individual chromosome structure while the trans-chromosomal subproblems represent how the chromosomes are organized in relation to each other within the nucleus. In the case of the fission yeast dataset six separate subproblems were generated: one for each chromosome's cis-interactions and one for each set of pairwise trans-chromosomal interactions (this division heuristic is described below). Each subproblem was independently run and the results were merged to generate the visualizations presented in Subsection 3.3. This is one of the first times a divide-and-conquer approach has been applied to 3D genome prediction from Hi-C data (cf. [48]).

\section{Divide}

A single whole-genome contact map can be naturally divided into a finite, organism specific number of subproblems representing its constituent cis-interactions and pairwise transinteractions. Each subproblem can be defined within the whole-genome contact map by specifying the range of genomic bins that correspond to the cis- or trans-interactions for each chromosome. In general, the number of subproblems for a whole-genome contact map with $k$ chromosomes is equal to $\frac{k(k-1)}{2}+k$ where $\frac{k(k-1)}{2}$ represents the number of pairwise trans-interaction subproblems and $k$ represents the number of cis-interaction subproblems. For example, because fission yeast has three chromosomes, its whole-genome contact map can be naturally partitioned into six subproblems (three cis- and three trans-interaction subproblems) to be solved in parallel. The location of these subproblems within a fission-yeast whole-genome contact map are depicted in Figure 4. 


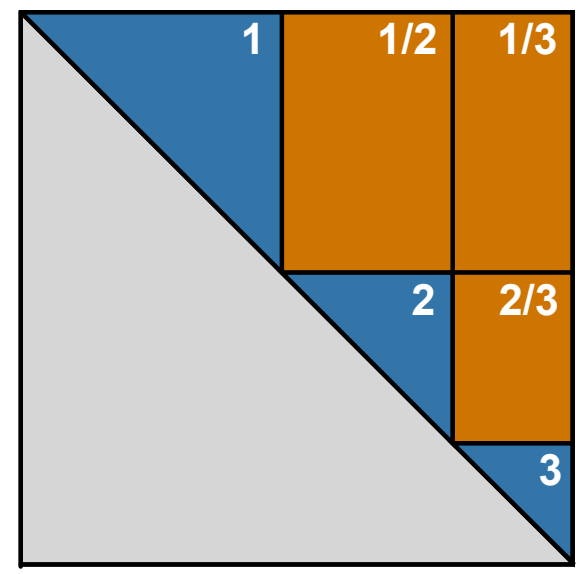

Figure 4: Identification of subproblems within the fission yeast contact map. The large grey triangle represents the portion of the contact map that does not need to be processed since all contact maps are mirrored along the diagonal. The blue triangles represent the subsections of the contact map that correspond to intra-chromosomal interactions, while the orange squares represent the subsections of the contact map that correspond to the inter-chromosomal interactions. The labels on the blue and orange areas represent the chromosome(s) involved in the interactions within that subsection of the contact map. In terms of the intra-chromosomal interactions, chromosome 1 contains the largest number of genomic bins while chromosomes 2 and 3 account for 34 and 80 percent fewer bins, respectively.

\section{Local Conquer}

In order to solve the entire 3D-GRP, programs corresponding to the cis-interaction subproblems and pairwise trans-interaction subproblems can be generated and run independently. The results can then be combined using the merge step described below.

\section{Merge}

The solutions from each subproblem are combined to reconstruct the entire 3D genomic model. This step is a heuristic which utilizes a novel coefficient (called the "dynamics coefficient") to account for the instances when a single genomic region participates in more than $m$ subproblem solutions; i.e. more than $m$ interactions. Instead of eliminating interactions from subproblem solutions involving the same genomic region (when this region has already been selected in $m$ interactions), each identified interaction is maintained and associated 
with a region-specific dynamics coefficient to encode the mobility (or lack of mobility) of that genomic region. Briefly, the dynamics coefficient for each genomic region is calculated by scanning all of the resultant files for each subproblem and counting how many times a specific genomic bin is found across the subproblem solution sets. The more interactions a genomic region is involved in, the higher its corresponding dynamics coefficient, and vice versa. In general, the dynamics coefficient is an integer value in the range of 0 to $k$ where $k$ is the number of chromosomes present in the genome. For example, in fission yeast $(k=3)$ if genomic bin 1 was involved in an interaction in the solution sets of the chromosome 1 cis-interaction subproblem and the chromosome 1/2 trans-interaction subproblem it would have a dynamics coefficient of 2 , whereas if it was involved in an interaction in each of the relevant trans-interaction subproblems and the cis-interaction subproblem it would have an associated dynamics coefficient of 3. A higher dynamics coefficient suggests that the corresponding genomic region was more mobile within the genome and that there was less certainty about its fixed position within the model. This is similar to the B factor (also known as the temperature factor or the Debye-Waller factor) generated with protein X-Ray Crystallography experiments [32]. The B factor encodes the degree of uncertainty associated with computed atomic positions in 3D space.

Utilizing the dynamics coefficient allows for the overall solution to the 3D-GRP to retain the information associated with each subproblem's optimal solution instead of having to exclude interactions that involve genomic regions already selected $m$ times as part of the solution set. Although this violates the initial ploidy restriction used to constrain the mathematical models, it is still biologically valid. As mentioned previously, it is possible for a given genomic bin to be involved in more than one interaction in 3D space [8, 18], even though $\mathrm{Hi}-\mathrm{C}$ is only able to detect one pairwise interaction per restriction site within a single haploid cell. Additionally, the dynamics coefficient allows the program to encode some of the mobility of genome organization into the predicted model by representing the certainty of whether an interaction is fixed within the population of cells. Finally, the dynamics co- 
efficient is also used to calculate relative distances between genomic bins which are used to visualize the predicted model.

\subsubsection{CP model}

The CP mathematical model (depicted in Mathematical Model 1) was implemented in MiniZinc [44] with the OR-Tools constraint solver from Google 22. An example MiniZinc program (Program 1) and a corresponding example data file (Example Data File 4) with the integral interaction frequencies from the hypothetical whole-genome contact map depicted in Figure 3A are given in the Appendix. This model leverages the fact that the solution will never contain more than $m \times N$ interactions making it scalable to larger genomes in terms of space complexity. It is worth noting that Equation (2) can be encoded by the inverse global constraint ${ }^{3}$, whereas Equation (1) is encoded with one element constraint per row of $A$ plus one sum constraint. These constraints are propagated by efficient algorithms in many constraint programming solvers.

The MiniZinc program corresponding to the complete fission yeast genome could not be solved to optimality after several days of run time on a server-grade computer. In an attempt to overcome this, the divide-and-conquer approach described above was applied. A MiniZinc program for each cis- or trans- subproblem was generated and run independently. Similarly to the complete whole-genome contact map, not a single cis- or trans-problem could be solved to optimality in several days.

\subsubsection{GM Model}

The GM mathematical model (depicted in Mathematical Model 2) was implemented in SICStus Prolog [4 [6] using Kolmogorov's Blossom V algorithm [28]. The implemented program using this representation is presented in Program 2. An example associated data

\footnotetext{
${ }^{2}$ https://developers.google.com/optimization/

3 http://www.minizinc.org/doc-lib/doc-globals-channeling.html

${ }^{4}$ http://sicstus.sics.se
} 
file for this program is given in Example Data File 5 and is based on the interaction frequency values from the hypothetical whole-genome contact map depicted in Figure 3A. The program is run by: (1) invoking the compile_adjacency predicate with a data file similar to that given in Example Data File 5 and (2) invoking the match_blossom5 predicate. In this example, this would be done by invoking: compile_adjacency('testMap.csv', testMap), followed by match_blossom5 (testMap, [1], [1]). For the fission yeast results, all of this has been automated in a "makefile" that is available on the project homepage (https://github.com/kimmackay/SonHi-C).

The SICStus Prolog implementation of the GM mathematical model was able to predict a fission yeast genomic organization in 1.088 seconds $(m=1$; for the complete wholegenome contact map where $|V|=1258,|E|=745595)$. In this matching, only one edge representing a trans-chromosomal interaction was included while the rest of the edges depicted cis-chromosomal interactions. This made it difficult to infer the organization of the chromosomes in relation to each other. In order to overcome this the divide-and-conquer approach described above was applied. Specifically, six separate matchings were identified: one for each chromosome's cis-interactions and one for each set of pairwise trans-chromosomal interactions. A SICStus Prolog program for each cis- or trans- subproblem was run independently. For each subproblem, the time it took to identify the optimal solution is presented in Table 1. These results were merged using the generate_gephi_input_subproblems.pl script available at the project homepage. The merge step took less than 1 second of execution time.

\subsubsection{IP Model}

The IP mathematical model (depicted in Mathematical Model 3) was implemented in Prolog and solved using the mixed integer programming based Gurobi Optimizer 55 [25]. The implemented program using this representation with the hypothetical whole-genome

\footnotetext{
5 http://www.gurobi.com/
} 


\begin{tabular}{|c|c|c|c|}
\hline Subproblem & $\begin{array}{c}\text { Number of } \\
\text { Vertices }(|V|)\end{array}$ & $\begin{array}{l}\text { Number of Edges } \\
(|E|)\end{array}$ & $\begin{array}{c}\text { Local Conquer } \\
\text { Runtime } \\
\text { (seconds) }\end{array}$ \\
\hline $\begin{array}{l}\text { chromosome } 1 \\
\text { cis-interaction }\end{array}$ & 558 & 148734 & 0.164 \\
\hline $\begin{array}{l}\text { chromosome } 2 \\
\text { cis-interaction }\end{array}$ & 454 & 96562 & 0.055 \\
\hline $\begin{array}{l}\text { chromosome } 3 \\
\text { cis-interaction }\end{array}$ & 246 & 27255 & 0.008 \\
\hline $\begin{array}{l}\text { chromosome } 1 / 2 \\
\text { trans-interaction }\end{array}$ & 454 & 241022 & 9.645 \\
\hline $\begin{array}{l}\text { chromosome } 1 / 3 \\
\text { trans-interaction }\end{array}$ & 246 & 128472 & 7.203 \\
\hline $\begin{array}{l}\text { chromosome } 2 / 3 \\
\text { trans-interaction }\end{array}$ & 246 & 103550 & 1.552 \\
\hline
\end{tabular}

Table 1: Subproblem sizes and corresponding run times for the GM mathematical model applied to the fission yeast whole-genome contact map.

contact map depicted in Figure $3 \mathrm{~A}$ is shown in Program 3. This implementation uses the same data file as the GM model (Example Data File 5). The program is run by: (1) invoking the compile_adjacency predicate with a data file similar to that given in Example Data File 5 and (2) invoking the solve_ip predicate. For this example, this would be done by invoking: compile_adjacency ('testMap.csv', testMap), followed by solve_ip(testMap, [1], [1]). For the fission yeast results, all of this has been automated in a makefile that is available on the project homepage (https://github.com/kimmackay/ SonHi-C).

The Prolog program for the complete whole-genome fission yeast contact map was able to predict a genomic organization in 294.44 seconds $(m=1 ;|V|=1258,|E|=745595)$. 
Similarly to the GM models' solution, only one trans-chromosomal interaction was represented in the solution set. The same trans-chromosomal interaction was present in the GM and IP solutions. The presence of only one trans-chromosomal interaction made it difficult to infer the organization of the chromosomes in relation to each other. In order to overcome this, the divide-and-conquer approach described above was used. Six separate subprograms were generated and run independently (one for each chromosome's cis-interactions and one for each set of pairwise trans-chromosomal interactions). The size of each problem in terms of $V$ and $E$ as well as the time it took to identify the optimal solution is presented in Table 2. In each case, the optimal solution identified was identical to the matching reported by the GM model. These results were merged using the generate_gephi_input_subproblems.pl script available at the project homepage. The merge step took less than 1 second of execution time.

\subsection{Visualization}

Since the results for the GM and IP models were identical, only one visualization is shown here. The results were converted into an undirected graph and visualized using Gephi (Figure 5A) [53, 54]. These images are graph-based visualizations of the predicted model based on the graphical representation of $\mathrm{Hi}-\mathrm{C}$ data described in GrapHi-C [37]. Briefly, the nodes in the network represent the individual genomic bins of the whole-genome contact map and the edges represent either selected interactions between bins or known linear interactions between adjacent bins. Linear interactions add additional biological constraints by representing the bonafide in vivo linear connections between bins (i.e. the linear extent of the chromosome). Each edge was weighted using either: the interaction frequency divided by the dynamics coefficient (for cis- and trans-interactions) or the experimental resolution (for linear interactions). The Force Atlas 2 layout was then applied to the network and the nodes were coloured according to their chromosome number or genomic feature. We would like to stress that this graph-based visualization is not a polymer model of the DNA chain that is 


\begin{tabular}{|c|c|c|c|}
\hline Subproblem & $\begin{array}{c}\text { Number of } \\
\text { Vertices }(|V|)\end{array}$ & $\begin{array}{l}\text { Number of Edges } \\
(|E|)\end{array}$ & $\begin{array}{c}\text { Local Conquer } \\
\text { Runtime } \\
\text { (seconds) }\end{array}$ \\
\hline $\begin{array}{l}\text { chromosome } 1 \\
\text { cis-interaction }\end{array}$ & 558 & 148734 & 15.75 \\
\hline $\begin{array}{l}\text { chromosome } 2 \\
\text { cis-interaction }\end{array}$ & 454 & 96562 & 9.26 \\
\hline $\begin{array}{l}\text { chromosome } 3 \\
\text { cis-interaction }\end{array}$ & 246 & 27255 & 2.06 \\
\hline $\begin{array}{l}\text { chromosome } 1 / 2 \\
\text { trans-interaction }\end{array}$ & 454 & 241022 & 4.90 \\
\hline $\begin{array}{l}\text { chromosome } 1 / 3 \\
\text { trans-interaction }\end{array}$ & 246 & 128472 & 4.70 \\
\hline $\begin{array}{l}\text { chromosome } 2 / 3 \\
\text { trans-interaction }\end{array}$ & 246 & 103550 & 3.40 \\
\hline
\end{tabular}

Table 2: Subproblem sizes and corresponding run times for the IP mathematical model applied to the fission yeast whole-genome contact map.

often seen in other $3 \mathrm{D}$ genome prediction tools. Therefore, the smoothness of the edges is not a result of any bending rigidity constraints. Instead, it is a result of the visualization tool (Gephi) and the network layout applied.

One of the most well-documented features of fission yeast genomic organization is the 3D clustering of centromeres and telomeres within the nucleus [9, 19]. In order to determine whether the predicted yeast model was able to recapitulate these features, the genomic bins corresponding to centromeres and telomeres were coloured in the Gephi visualization. Figure $5 \mathrm{~B}$ provides a visual depiction of the location of the centromeres and telomeres in the predicted genomic model. This figure provides further evidence that the predicted genome model is consistent with established principles of mitotic fission yeast chromosomal orga- 


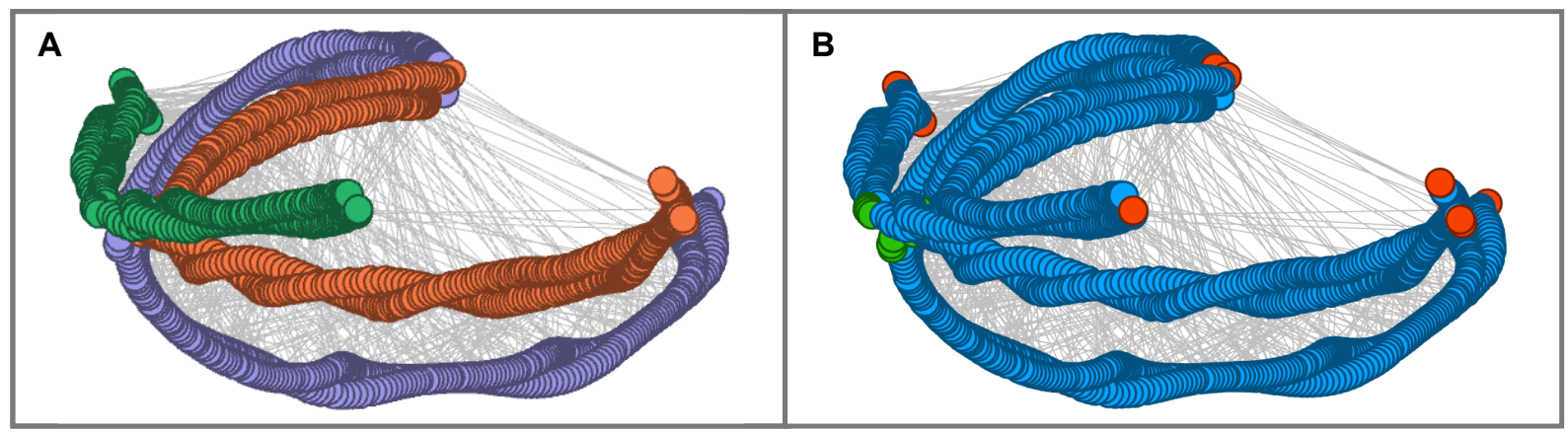

Figure 5: Visualization of the Predicted Genome Model Using the GM/IP Models and Identification of Genomic Features Indicative of Fission Yeast Mitotic Chromosomal Organization. Circles depict the genomic bins, grey lines represent cis- and trans-interaction edges selected by the GM/IP models, and line lengths are proportional to the associated dynamics coefficient and the inverse of the interaction frequency $\left(A_{i, j}\right)$. In Panel $\mathrm{A}$, circles are coloured according to their corresponding chromosome (CHR1: purple, CHR2: orange, CHR3: green). In Panel B, the following genomic features are highlighted: telomeres (red), centromeres (green) and nuclear DNA (blue).

nization including: (1) chromosomal organization into a hemispherical region, (2) a single centromere cluster and (3) the presence of two telomere clusters (chromosome 1/2) located near the nuclear periphery, opposite the centromere cluster [55]. The clustering of the regions appears to be conserved in the predicted model providing confidence in the biological accuracy that was achieved using the $\mathbf{G M}$ and IP mathematical models and the corresponding non-procedural implementations.

\subsection{Effect of $m$ on Genome Organization in Fission Yeast}

As mentioned previously, it is possible that each genomic region could be involved with more than one interaction within the genome but is restricted to $m \mathrm{Hi}-\mathrm{C}$ interactions (where the value of $m$ is based on organism ploidy). To determine whether or not relaxing this ploidy restriction would result in a more comprehensive genomic model, the implemented program for the IP model was tested with values of $m$ from 1 to 6 for the same fission yeast Hi-C dataset used above (GSM1379427 [42]). As mentioned previously, this mathematical model 
allows for a single genomic bin to be involved in more than one Hi-C mediated interaction in the predicted genome organization. For each value of $m$, the program was able to find an optimal solution in 294.44 seconds, 13.20 seconds, 104.46 seconds, 15.31 seconds, 38.79 seconds, and 16.94 seconds for $m=1 . .6$, respectively. Similarly to what was described above, the results for each value of $m$ were converted into a graph and visualized using Gephi (Figure 6). Each edge was weighted using either: the interaction frequency divided by the dynamics coefficient (for cis- and trans-interactions) or the experimental resolution (for linear interactions). The Force Atlas 2 layout was then applied to the network and the nodes were coloured according to their chromosome number. The nodes in the graph represent the individual genomic bins of the whole-genome contact map and the edges represent either selected interactions between bins or known linear interactions between adjacent bins.

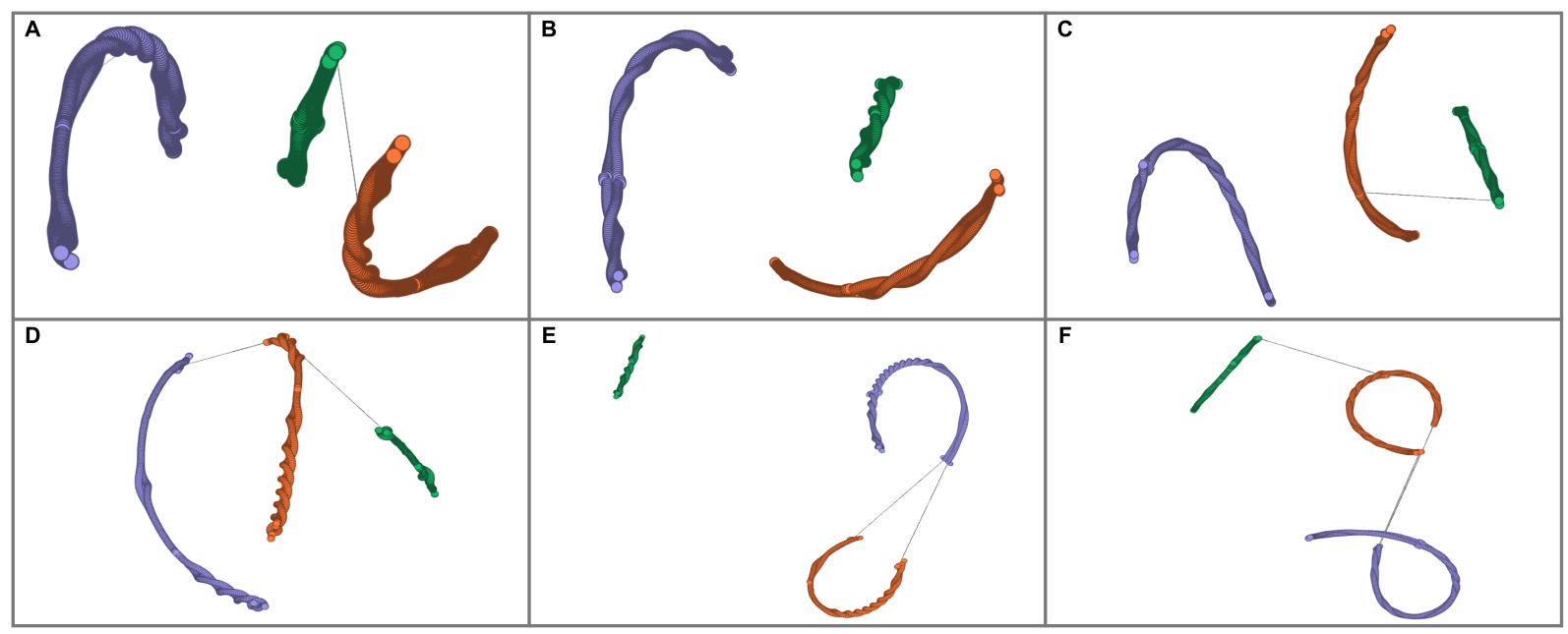

Figure 6: Visualization of the Predicted Genome Model Using the IP Model with Various $m$ Values. Circles depict the genomic bins, grey lines represent trans-interaction edges selected by the IP model, and line lengths are proportional to the associated interaction frequency $\left(A_{i, j}\right)$. Circles are coloured according to their corresponding chromosome (CHR1: purple, CHR2: orange, CHR3: green). The results for each $m$ values are presented in the following panels: A $(m=1), \mathrm{B}(m=2), \mathrm{C}(m=3), \mathrm{D}(m=4), \mathrm{E}(m=5), \mathrm{F}(m=6)$.

The results presented in Figure 6 indicate that relaxing the ploidy restriction (by increasing the value of $m$ ) does not result in a more comprehensive genomic model. Similarly to 
the GM and IP whole-genome predictions, minimal trans-chromosomal interactions were selected by the model regardless of what the parameter $m$ was set to. Specifically the following number of trans-chromosomal interactions were observed in each solution set: $1(m=1)$, $0(m=2), 1(m=3), 2(m=4), 2(m=5), 3(m=6)$. It is clear that regardless of the number of interactions allowed per genomic bin, separating the 3D-GRP into cis- and transsubproblems is a more viable strategy for predicting genome organization when using the IP mathematical model. This is likely due to the fact that cis-chromosomal interactions occur more frequently than trans-chromosomal interactions within the genome (resulting in higher interaction frequency values) [12].

\subsection{Application to Organisms with Higher Ploidies and/or Larger Genomes}

The IP mathematical model described above could be easily applied to organisms with higher ploidies. As mentioned previously, the $m$ parameter defines the number of Hi-C mediated interactions in which each genomic bin can actively be participating within the solution set. Therefore, this model could be easily applied to organisms with higher ploidies by specifying the value of the $m$ parameter. For instance, $m$ could be modified in the following ways according to the number of chromosome copies present: $m=2$ (diploid; common in mammals), $m=4$ (tetraploid; common in plants), and so on. One issue that would need to be addressed in organisms with higher ploidies is phasing the interactions to each chromosome copy. This could potentially be solved using existing phasing tools [10] and additional biological data [4, 51].

Utilizing the divide-and-conquer approach described in Section 3.2 allows one to take advantage of coarse-grained parallelism ensuring the mathematical models are scaleable to organisms with larger genomes (for $m \geq 1$ ). This type of parallelism is easy to obtain on many types of computational infrastructure. As an example, it could be easily applied to a whole-genome contact map from Homo sapiens. This contact map would result in the 
generation of 276 subproblems (given $k=23$ and the number of subproblems $=\frac{k(k-1)}{2}+k$ ). It would not be hard to find 276 cores in the current environment of computer grids and multi-core machines to run the problems representing the subproblems in parallel. Since each subproblem can be run in parallel and the merge step has a linear time complexity (in terms of the number of genomic bins), the associated average runtime of the computation is expected to be polynomial in the number of genomic bins. In general the complexity will depend on the chromosome size and the associated experimental resolution).

\section{Future Work}

Future work will focus on the validation, modification and extension of the 3D-GRP solutions presented in this manuscript. Specifically, an extensive biological validation of the predicted genome models will be performed to better characterize the biological accuracy of the developed mathematical models. The formulation for the $\mathbf{C P}$ mathematical model will be modified to adjust the proportion of cis-and trans-chromosomal interactions in the solution set. Additionally, different types of data transformations will be investigated to better accommodate the proportion of cis-and trans-chromosomal interactions in the whole-genome contact map. The GM model will be parameterized so that it can be used with different values of $m$. The IP mathematical model will be utilized as a computational framework which will be extended and further developed to incorporate a variety of additional genomic datasets and information types into the prediction process. The non-procedural representation utilized in this computational framework will allow additional datasets to be naturally incorporated into the prediction of the 3D genome. For example, each genomic bin could have an associated list of variables representing the genes found within that bin and their corresponding gene expression values. Constraints could then be applied to favour interactions between regions with similar expression profiles. The IP mathematical model will also be utilized as a starting point for predicting the 3D genomic structure of organisms with 
higher ploidies by applying the modifications suggested in the subsection above. Finally, the IP model could be adapted and used for detecting topologically associated domains [13, 22] within a whole-genome contact map.

\section{Conclusion}

This is the first time a non-procedural programming approach has been used to model the 3D genome organization from Hi-C data. Specifically, we developed three novel mathematical models for predicting the 3D genome from Hi-C data. Each mathematical model was implemented in a non-procedural language and tested with an existing Hi-C dataset from fission yeast. The $\mathbf{C P}$ model was not able to solve the 3D-GRP to optimality after several days of execution. The GM model was able to optimally solve the 3D-GRP for fission yeast in 1.088 seconds. The IP model was able to optimally solve the 3D-GRP in fission yeast for $m=1$ in 294.44 seconds resulting in the same solution as the GM model. In this solution, only one trans-chromosomal interaction was selected making it difficult to infer the organization of the chromosomes in relation to each other. A divide-and-conquer approach was used to overcome this where six separate subproblems (one for each set of cis-chromosomal interactions and one for each set of pairwise trans-chromosomal interactions) were independently solved, combined and visualized. The cis-chromosomal matchings better represent individual chromosome structure while the trans-chromosomal matchings represent how the chromosomes are organized in relation to each other within the nucleus. In the case of the GM and IP models, the predicted genome organizations represent the interactions of a population-averaged consensus structure where the most likely interactions are present (this is determined by maximizing the sum of the interaction frequencies of the selected interactions). Each predicted genome organization was then biologically validated through literature search which verified that the prediction recapitulated key documented features of the yeast genome. The divide-and-conquer solution strategy lends itself to ad- 
ditional speed improvements due to the potential for running the defined subproblems in parallel. Furthermore, a novel coefficient was defined (the dynamics coefficient) which allowed a level of positional uncertainty to be encoded into the predicted genomic organization. Overall, the mathematical models and programs developed here demonstrate the power of non-procedural applications for modelling the 3D genome and are a step towards a better understanding of the relationship between genomic structure and function.

\section{Declarations}

\section{Ethics approval and consent to participate}

Not applicable

\section{Consent for publication}

Not applicable.

\section{Availability of data and material}

The datasets supporting the conclusions of this article are available in the Gene Expression Omnibus database(accession number: GSM1379427; https://www.ncbi.nlm.nih.gov/geo/ query/acc . cgi?acc=GSM1379427).

\section{Software information}

Project Name: SonHi-C (pronounced "sonic")

Project home page: https://github.com/kimmackay/SonHi-C

License: This work is licensed under the Creative Commons Attribution-NonCommercialShareAlike 3.0 Unported License. To view a copy of this license, visit http://creativecommons . org/licenses/by-nc-sa/3.0/ or send a letter to Creative Commons, PO Box 1866, Mountain View, CA 94042, USA. 


\section{Competing interests}

The authors declare that they have no competing interests.

\section{Funding}

This work was supported by the Natural Sciences and Engineering Research Council of Canada [RGPIN 37207 to AK, Vanier Canada Graduate Scholarship to KM].

\section{Author's contributions}

KM, MC, AK developed the CP and GM mathematical models. MC developed the IP mathematical model. MC implemented and tested the constraint models. KM visualized the results and verified the biological accuracy. KM wrote the manuscript. MC, AK edited the manuscript.

\section{Acknowledgements}

We could like to thank Dr. Christopher Eskiw and Conor Lazarou for their input and advice.

\section{Authors' information}

The e-mail address for each author is as follows: Kimberly MacKay (kimberly.mackay@usask.ca); Mats Carlsson (mats.carlsson@ri.se); Anthony Kusalik (kusalik@cs.usask.ca).

\section{References}

[1] Ferhat Ay, Evelien M. Bunnik, Nelle Varoquaux, Sebastiaan M. Bol, Jacques Prudhomme, Jean-Philippe Vert, William Stafford Noble, and Karine G. Le Roch. Threedimensional modeling of the $P$. falciparum genome during the erythrocytic cycle re- 
veals a strong connection between genome architecture and gene expression. Genome Research, 24:974-988, March 2014.

[2] Ferhat Ay and William S. Noble. Analysis methods for studying the 3D architecture of the genome. Genome Biology, 16(1):1-15, September 2015.

[3] Sepideh Babaei, Waseem Akhtar, Johann de Jong, Marcel Reinders, and Jeroen de Ridder. 3D hotspots of recurrent retroviral insertions reveal long-range interactions with cancer genes. Nature Communications, 6:6381, 2015.

[4] Shay Ben-Elazar, Benny Chor, and Zohar Yakhini. Extending partial haplotypes to full genome haplotypes using chromosome conformation capture data. Bioinformatics, 32:i559-i566, 2016.

[5] Federico Campeotto, Alessandro Dal Palù, Agostino Dovier, Ferdinando Fioretto, and Enrico Pontelli. A constraint solver for flexible protein models. Journal of Artificial Intelligence Research, 48:93-1000, 2013.

[6] Mats Carlsson and Per Mildner. Sicstus prolog - the first 25 years. Theory and Practice of Logic Programming, 12(1-2):35-66, 2012.

[7] Lyubomira Chakalova, Emmanuel Debrand, Jennifer A. Mitchell, Cameron S. Osborne, and Peter Fraser. Replication and transcription: shaping the landscape of the genome. Nature Reviews Genetics, 6(9):669-677, 2005.

[8] Andrea M. Chiariello, Carlo Annunziatella, Simona Bianco, Andrea Esposito, and Mario Nicodemia. Polymer physics of chromosome large-scale 3D organisation. Scientific Reports, 6:29775, July 2016.

[9] Yuji Chikashige, Da-Qiao Ding, Yoshiyuki Imai, Masayuki Yamamoto, Tokuko Haraguchi, and Yasushi Hiraoka. Meiotic nuclear reorganization: switching the po- 
sition of centromeres and telomeres in the fission yeast Schizosaccharomyces pombe. The EMBO Journal, 16(1):193-202, 1997.

[10] Yongwook Choi, Agnes P. Chan, Ewen Kirkness, Amalio Telenti, and Nicholas J. Schork. Comparison of phasing strategies for whole human genomes. PLOS Genetics, 14(4):e1007308, 2018.

[11] Job Dekker. Regulation of gene expression through chromatin interaction networks. Blood Cells, Molecules, and Diseases, 38(2):135, 2007.

[12] Job Dekker and Leonid Mirny. The 3D genome as moderator of chromosomal communication. Cell, 164(6):1110-1121, 2016.

[13] Jesse R Dixon, Siddarth Selvaraj, Feng Yue, Audrey Kim, Yan Li, Yin Shen, Ming Hu, Jun S Liu, and Bing Ren. Topological domains in mammalian genomes identified by analysis of chromatin interactions. Nature, 485(7398):376-380, 2012.

[14] Zhijun Duan, Mirela Andronescu, Kevin Schutz, Sean McIlwain, Yoo Jung Kim, Choli Lee, Jay Shendure, Stanley Fields, C. Anthony Blau, and William S. Noble. A threedimensional model of the yeast genome. Nature, 465:363-367, 2010.

[15] Jack Edmonds. Paths, trees, and flowers. Canadian Journal of mathematics, 17(3):449$467,1965$.

[16] Damien Eveillard, Delphone Ropers, Hidde de Jong, Christiane Branlant, and Alexander Bockmayr. A multi-scale constraint programming model of alternative splicing regulation. Computational Systems Biology, 325(1):3-24, 2004.

[17] Eric Fanchon, Fabien Corblin, Laurent Trilling, Bastien Hermant, and Danielle Gulino. Modeling the molecular network controlling adhesion between human endothelial cells: Inference and simulation using constraint logic programming. In Vincent Danos and 
Vincent Schachter, editors, Computational Methods in Systems Biology, volume 3082 of Lecture Notes in Computer Science, pages 104-118. Springer Berlin Heidelberg, 2005.

[18] Stephanie Fanucchi, Youtaro Shibayama, Shaun Burd, Marc S. Weinberg, and Musa M. Mhlanga. Chromosomal contact permits transcription between coregulated genes. Cell, 115(3):606-620, 2013.

[19] Hironori Funabiki, Iain Hagan, Satoru Uzawa, and Mitsuhiro Yanagida. Cell cycledependent specific positioning and clustering of centromeres and telomeres in fission yeast. Journal of Cell Biology, 121:961-976, June 1993.

[20] Juan Antonio Garcia-Martin, Ivan Dotu, Javier Fernandez-Chamorro, Gloria Lozano, Jorge Ramajo, Encarnacion Martinez-Salas, and Peter Clote. RNAiFold2T: Constraint programming design of thermo-IRES switches. Bioinformatics, 32(12):i360-i368, June 2016.

[21] Zoe E Gillespie, Kimberly MacKay, Michelle Sander, Brett Trost, Wojciech Dawicki, Aruna Wickramarathna, John Gordon, Mark Eramian, Ian R Kill, Joanna M Bridger, Anthony Kusalik, Jennifer A Mitchell, and Christopher H Eskiw. Rapamycin reduces fibroblast proliferation without causing quiescence and induces stat5a/b-mediated cytokine production. Nucleus, 6(6):490-506, 2015.

[22] Nathan Harmston, Elizabeth Ing-Simmons, Ge Tan, Malcolm Perry, Matthias Merkenschlager, and Boris Lenhard. Topologically associating domains are ancient features that coincide with metazoan clusters of extreme noncoding conservation. Nature Communications, 8:441, 2017.

[23] Ming Hu, Ke Deng, Zhaohui Qin, Jesse Dixon, Siddarth Selvaraj, Jennifer Fang, Bing Ren, and Jun S. Liu. Bayesian inference of spatial organizations of chromosomes. PLOS Computational Biology, 9(1):e1002893, January 2013. 
[24] Maxim Imakaev, Geoffrey Fudenberg, Rachel Patton McCord, Natalia Naumova, Anton Goloborodko, Bryan R. Lajoie, Job Dekker, and Leonid A. Mirny. Iterative correction of Hi-C data reveals hallmarks of chromosome organization. Nature Methods, 9(10):999$1003,2012$.

[25] Gurobi Optimization Inc. Gurobi Optimizer Reference Manual, 2014. http://www.gurobi.com.

[26] Joxan Jaffar and Michael J Maher. Constraint logic programming: a survey. Journal of Logic Programming, 19(20):503-581, 1994.

[27] Philip A. Knight and Daniel Ruiz. A fast algorithm for matrix balancing. IMA Journal of Numerical Analysis, 33(3):1029-1047, 2012.

[28] Vladimir Kolmogorov. Blossom V: a new implementation of a minimum cost perfect matching algorithm. Mathematical Programming Computation, 1(1):43-67, 2009.

[29] Ludwig Krippahl and Pedro Barahona. Applying constraint programming to protein structure determination. In Principles and Practice of Constraint Programming - CP'99, pages 289-302. Springer Berlin Heidelberg, 1999.

[30] Ludwig Krippahl and Pedro Barahona. PSICO: Solving protein structures with constraint programming and optimization. Constraints, 7(3):317-331, 2002.

[31] Masahiko Kuroda, Hideyuki Tanabe, Keiichi Yoshida, Kosuke Oikawa, Akira Saito, Tomoharu Kiyuna, Hiroshi Mizusawa, and Kiyoshi Mukai. Alteration of chromosome positioning during adipocyte differentiation. Journal of Cell Science, 117:5897-5903, November 2004.

[32] Antonija Kuzmanic, Navraj S. Pannu, and Bojan Zagrovic. X-ray refinement significantly underestimates the level of microscopic heterogeneity in biomolecular crystals. Nature Communications, 5:3220, 2014. 
[33] Bryan R Lajoie, Job Dekker, and Noam Kaplan. The hitchhiker's guide to Hi-C analysis: Practical guidelines. Methods, 72:65-75, January 2015.

[34] Annick Lesne, Julien Riposo, Paul Roger, Axel Cournac, and Julien Mozziconacci. 3D genome reconstruction from chromosomal contacts. Nature Methods, 11:1141-1143, March 2014.

[35] Wenyuan Li, Ke Gong, Qingjiao Li, Frank Alber, and Xianghong Jasmine Zhou. HiCorrector: a fast, scalable and memory-efficient package for normalizing large-scale Hi-C data. Bioinformatics, 31(6):960-962, 2015.

[36] Erez Lieberman-Aiden, Nynke L van Berkum, Louise Williams, Maxim Imakaev, Tobias Ragoczy, Agnes Telling, Ido Amit, Bryan R Lajoie, Peter J Sabo, Michael O Dorschner, Richard Sandstrom, Bradley Bernstein, M A Bender, Mark Groudine, Andreas Gnirke, John Stamatoyannopoulos, Leonid A Mirny, Eric S Lander, and Job Dekker. Comprehensive mapping of long range interactions reveals folding principles of the human genome. Science, 326(5950):289-293, October 2009.

[37] Kimberly MacKay, Anthony Kusalik, and Christopher H. Eskiw. GrapHi-C: graphbased visualization of Hi-C datasets. BMC Research Notes, 11(1):418, 2018.

[38] Kurt Mehlhorn and Guido Schäfer. Implementation of $O(n m \log n)$ weighted matchings in general graphs: the power of data structures. Journal of Experimental Algorithmics (JEA), 7:4, 2002.

[39] Ishita S Mehta, Manelle Amira, Amanda J Harvey, and Joanna M Bridger. Rapid chromosome territory relocation by nuclear motor activity in response to serum removal in primary human fibroblasts. Genome Biology, 11(1):R5, January 2010.

[40] Ishita S Mehta, Christopher H Eskiw, Halime D Arican, Ian R Kill, and Joanna M Bridger. Farnesyltransferase inhibitor treatment restores chromosome territory posi- 
tions and active chromosome dynamics in Hutchinson-Gilford progeria syndrome cells. Genome Biology, 12(8):R74, August 2011.

[41] Ishita S Mehta, Mugdha Kulashreshtha, Sandeep Chakraborty, Ullas KolthurSeetharam, and Basuthkar J Rao. Chromosome territories reposition during DNA damage-repair response. Genome Biology, 14(12):R135, December 2013.

[42] Takeshi Mizuguchi, Geoffrey Fudenberg, Sameet Mehta, Jon-Matthew Belton, Nitika Taneja, Hernan Diego Folco, Peter FitzGerald, Job Dekker, Leonid Mirny, Jemima Barrowman, and Shiv IS Grewal. Cohesin-dependent globules and heterochromatin shape 3D genome architecture in S. pombe. Nature, 516(7531):432-435, December 2014.

[43] Takashi Nagano, Yaniv Lubling, Tim J Stevens, Stefan Schoenfelder, Eitan Yaffe, Wendy Dean, Ernest D Laue, Amos Tanay, and Peter Fraser. Single-cell Hi-C reveals cell-to-cell variability in chromosome structure. Nature, 502:59-64, October 2013.

[44] Nicholas Nethercote, Peter J. Stuckey, Ralph Becket, Sebastian Brand, Gregory J. Duck, and Guido Tack. MiniZinc: Towards a standard CP modelling language. In Christian Bessière, editor, Principles and Practice of Constraint Programming - CP 200\%, LNCS, pages 529-543, Providence, RI, USA, September 23-27 2007. Springer Berlin Heidelberg.

[45] Luiz Fernando Nunes, Lauro Cesar Galvão, Heitor Silvério Lopes, Pablo Moscato, and Regina Berretta. An integer programming model for protein structure prediction using the 3D-HP side chain model. Discrete Applied Mathematics, 198(C):206-214, 2016.

[46] Alessandro Dal Palù, Agostino Dovier, and Federico Fogolari. Constraint logic programming approach to protein structure prediction. BMC Bioinformatics, 5:186, December 2004.

[47] Vladimir Reinharz, François Major, and Jérôme Waldispühl. Towards 3D structure 
prediction of large RNA molecules: an integer programming framework to insert local 3d motifs in rna secondary structure. Bioinformatics, 28(12):i207-i214, 2012.

[48] Lila Rieber and Shaun Mahony. miniMDS: 3D structural inference from high-resolution Hi-C data. Bioinformatics, 33(14):i261-i266, 2017.

[49] F. Rossi, P. van Beek, and T. Walsh, editors. Handbook of Constraint Programming. Elsevier, New York, NY, USA, 2006.

[50] Mark R Segal and Henrik L Bengtsson. Reconstruction of 3D genome architecture via a two-stage algorithm. BMC Bioinformatics, 16:373, November 2015.

[51] Siddarth Selvaraj, Jesse R Dixon, Vikas Bansal, and Bing Ren. Whole-genome haplotype reconstruction using proximity-ligation and shotgun sequencing. Nature Biotechnology, 31(12):1111-1118, 2013.

[52] François Serra, Marco Di Stefano andYannick G. Spill, Yasmina Cuartero, Michael Goodstadt, Davide Baù, and Marc A. Marti-Renom. Restraint-based three-dimensional modeling of genomes and genomic domains. FEBS Letters, 589(20):2987-2995, May 2015.

[53] Paul Shannon, Andrew Markiel, Owen Ozier, Nitin S. Baliga, Jonathan T. Wang, Daniel Ramage, Nada Amin, Benno Schwikowski, and Trey Ideker. Cytoscape: A software environment for integrated models of biomolecular interaction networks. Genome Research, 13(11):2498-2504, 2003.

[54] Michael E Smoot, Keiichiro Ono, Johannes Ruscheinski, Peng-Liang Wang, and Trey Ideker. Cytoscape 2.8: new features for data integration and network visualization. Bioinformatics, 27(3):431-432, February 2011.

[55] S Uzawa and M Yanagida. Visualization of centromeric and nucleolar DNA in fission 
yeast by fluorescence in situ hybridization. Journal of Cell Science, 101(Pt 2):267-275, 1992.

[56] Nelle Varoquaux, Ferhat Ay, William Stafford Noble, and Jean-Philippe Vert. A statistical approach for inferring the 3D structure of the genome. Bioinformatics, 30(12):i26i33, 2014.

[57] Laurence A Wolsey. Integer Programming. Wiley, 1998.

[58] Mitsuhiro Yanagida. The model unicellular eukaryote, Schizosaccharomyces pombe. Genome Biology, 3(3), February 2002.

[59] Jason Yao, Vladimir Reinharz, François Major, and Jérôme Waldispühl. RNA-MoIP: prediction of RNA secondary structure and local 3D motifs from sequence data. Nucleic Acids Research, 45(W1):W440-W444, 2017. 


\section{A Implemented Programs}

Program 1: The MiniZinc implementation of the CP mathematical model.

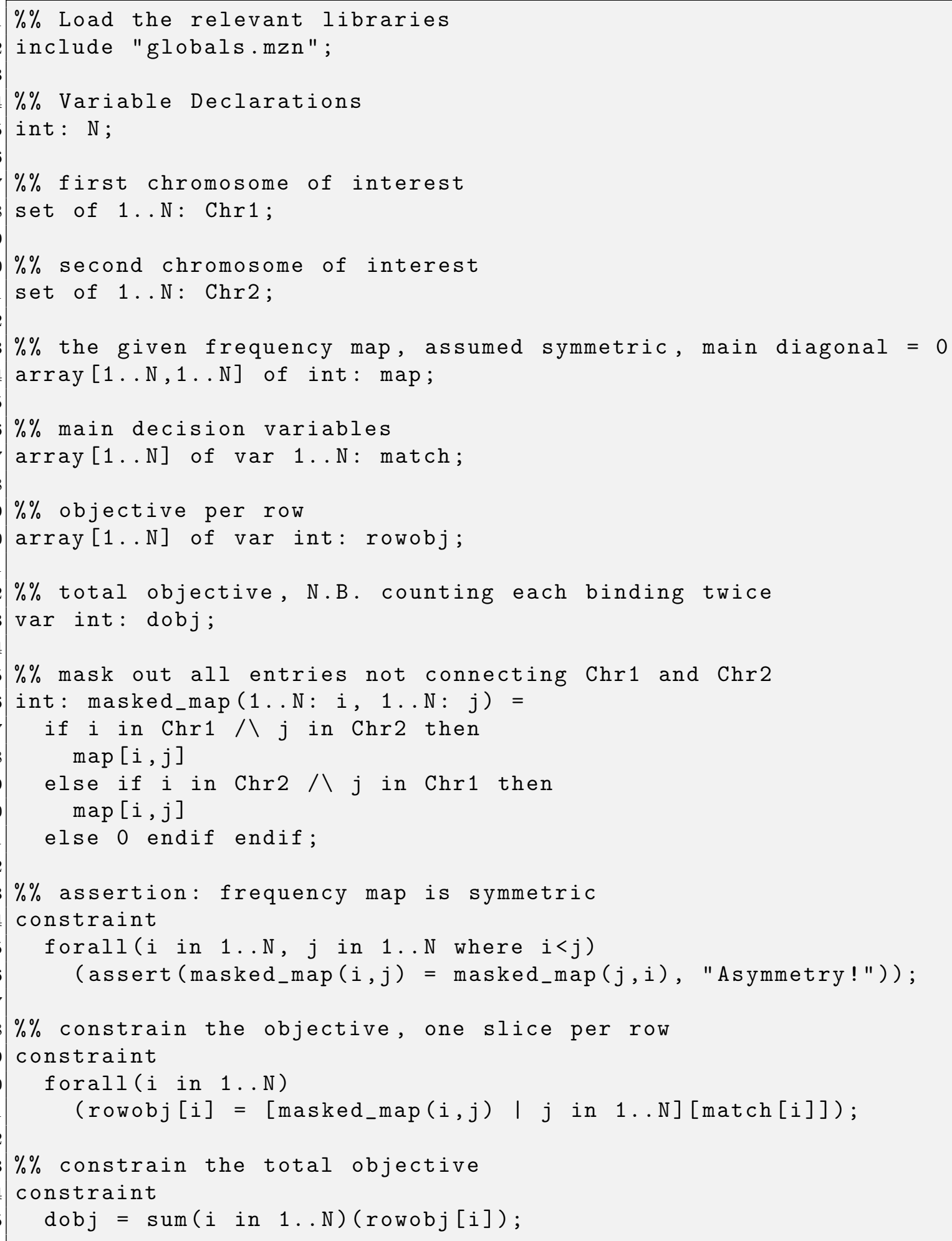




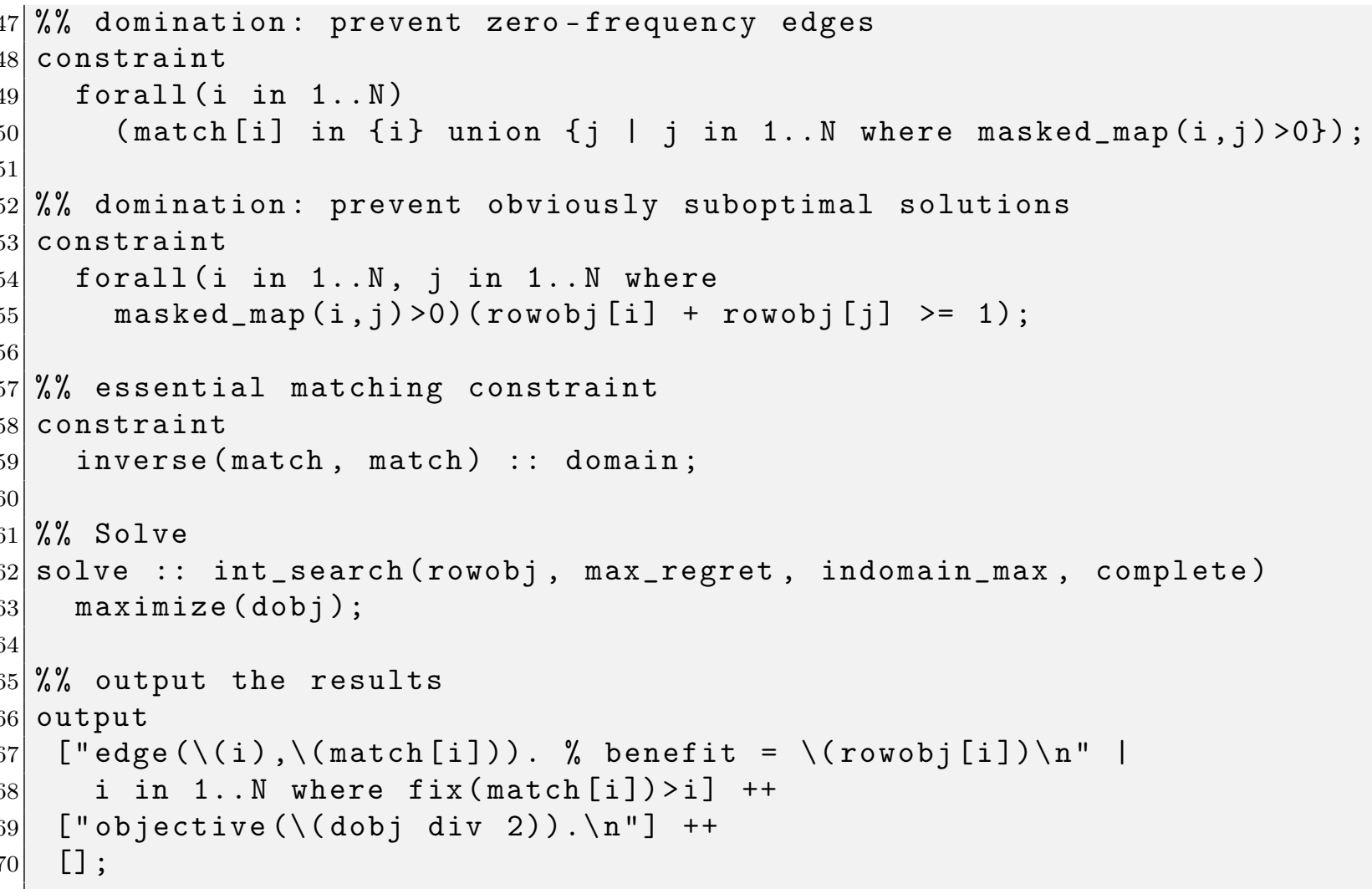

Program 2: The implemented program using the GM mathematical model.

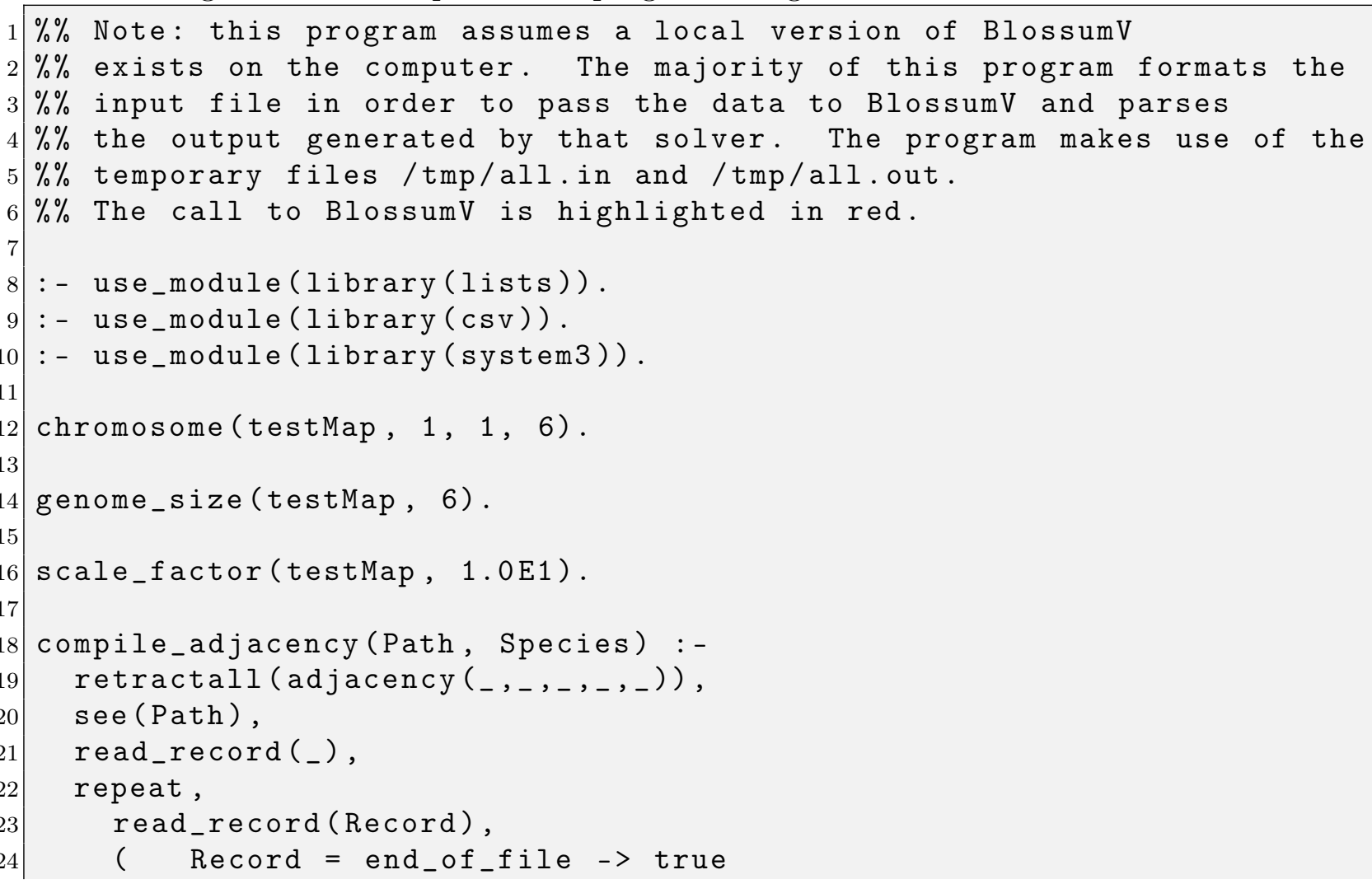




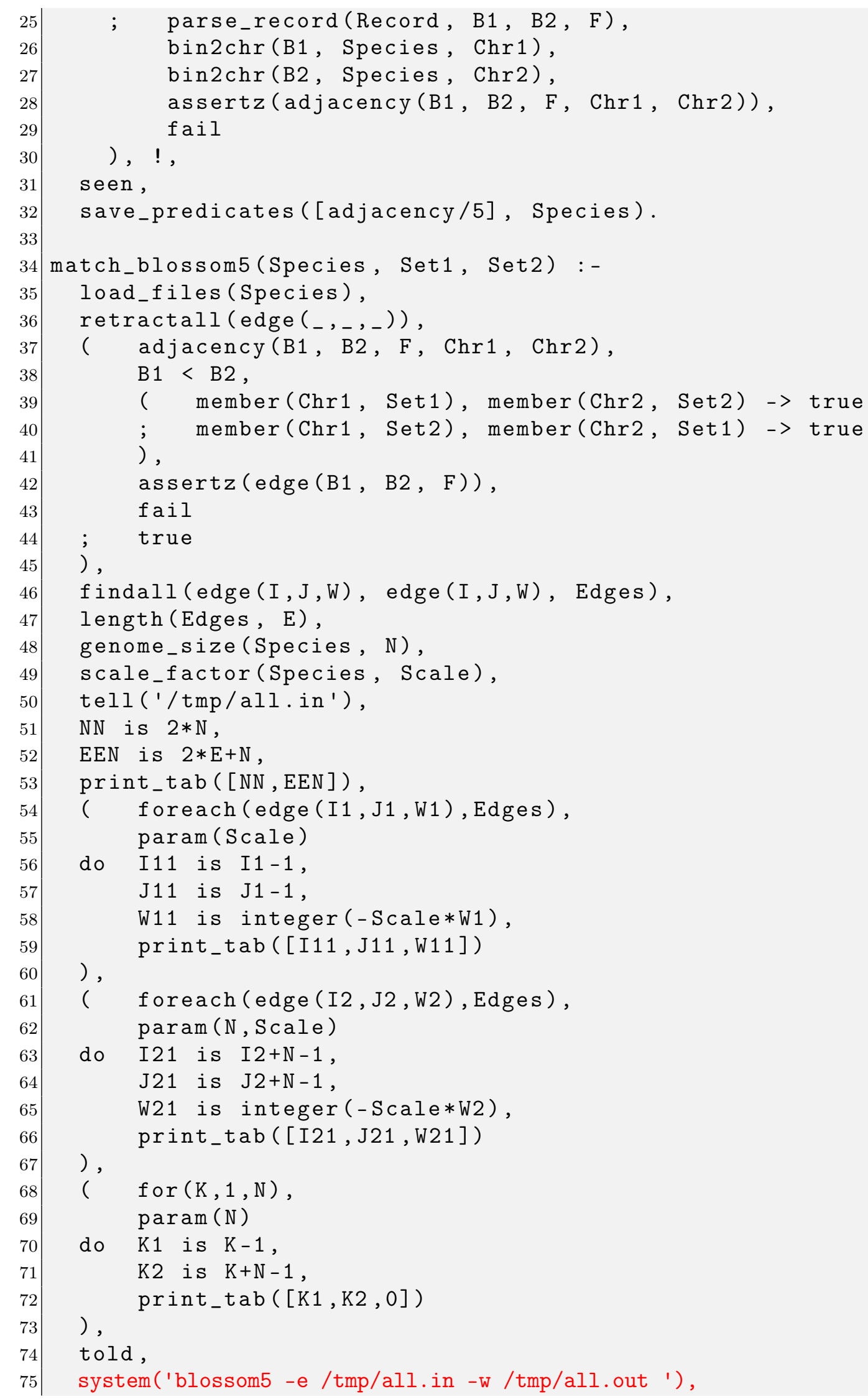




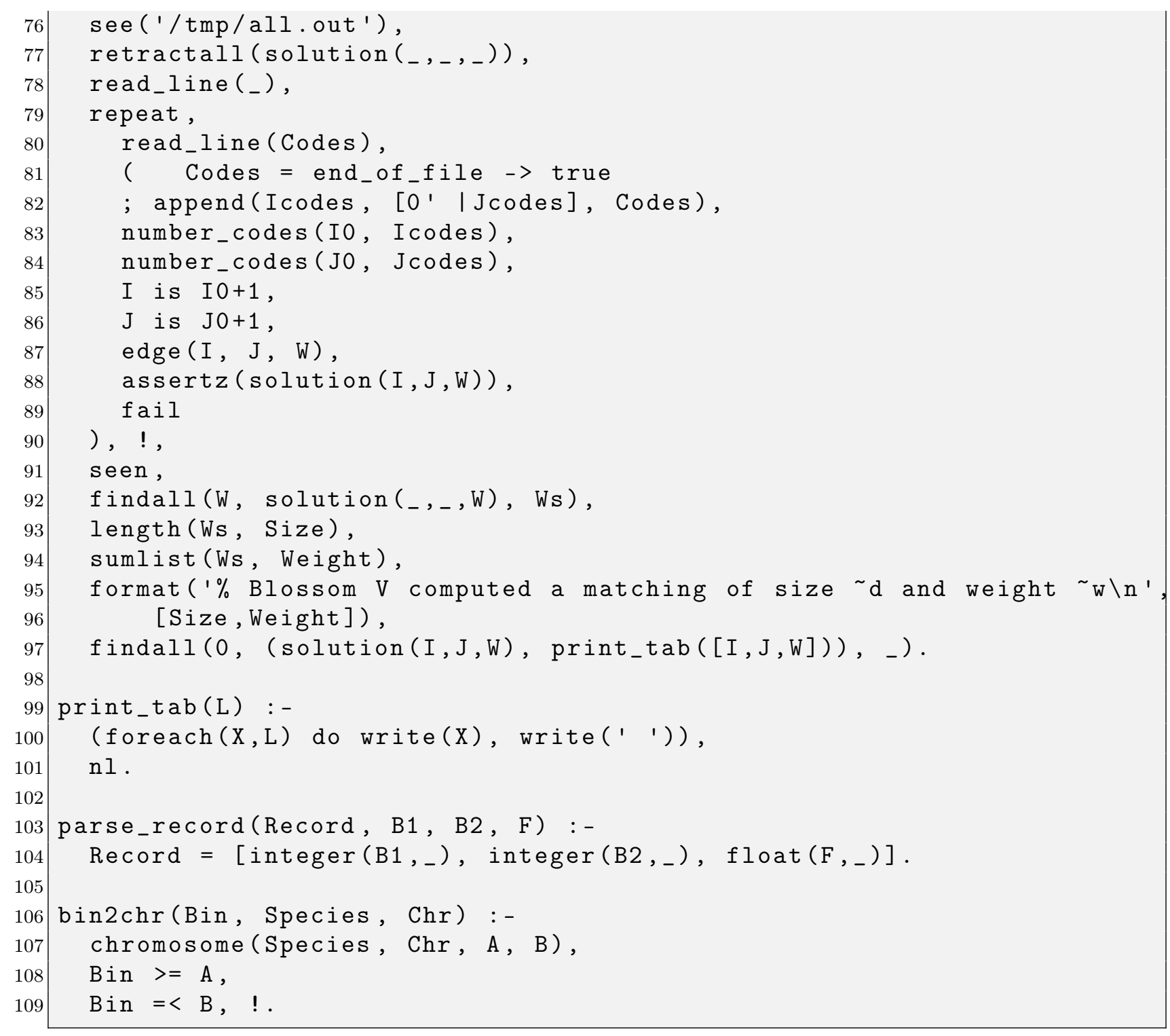

Program 3: The implemented program using the IP mathematical model.

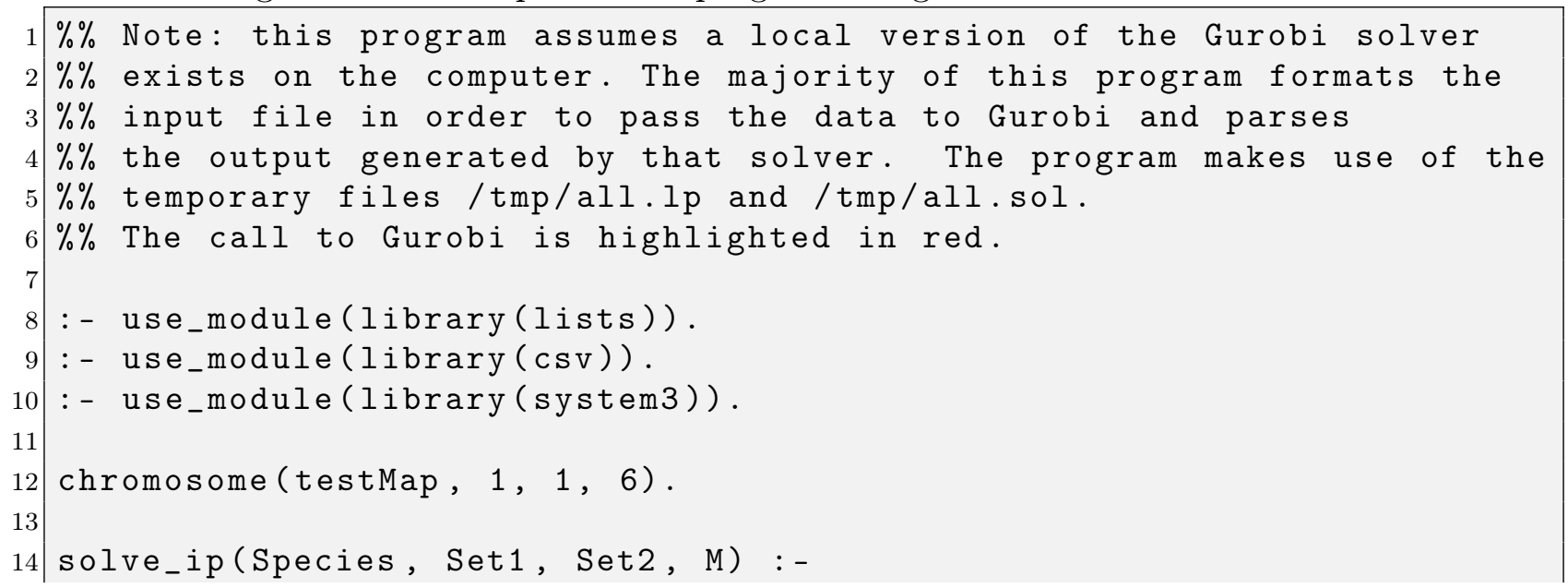




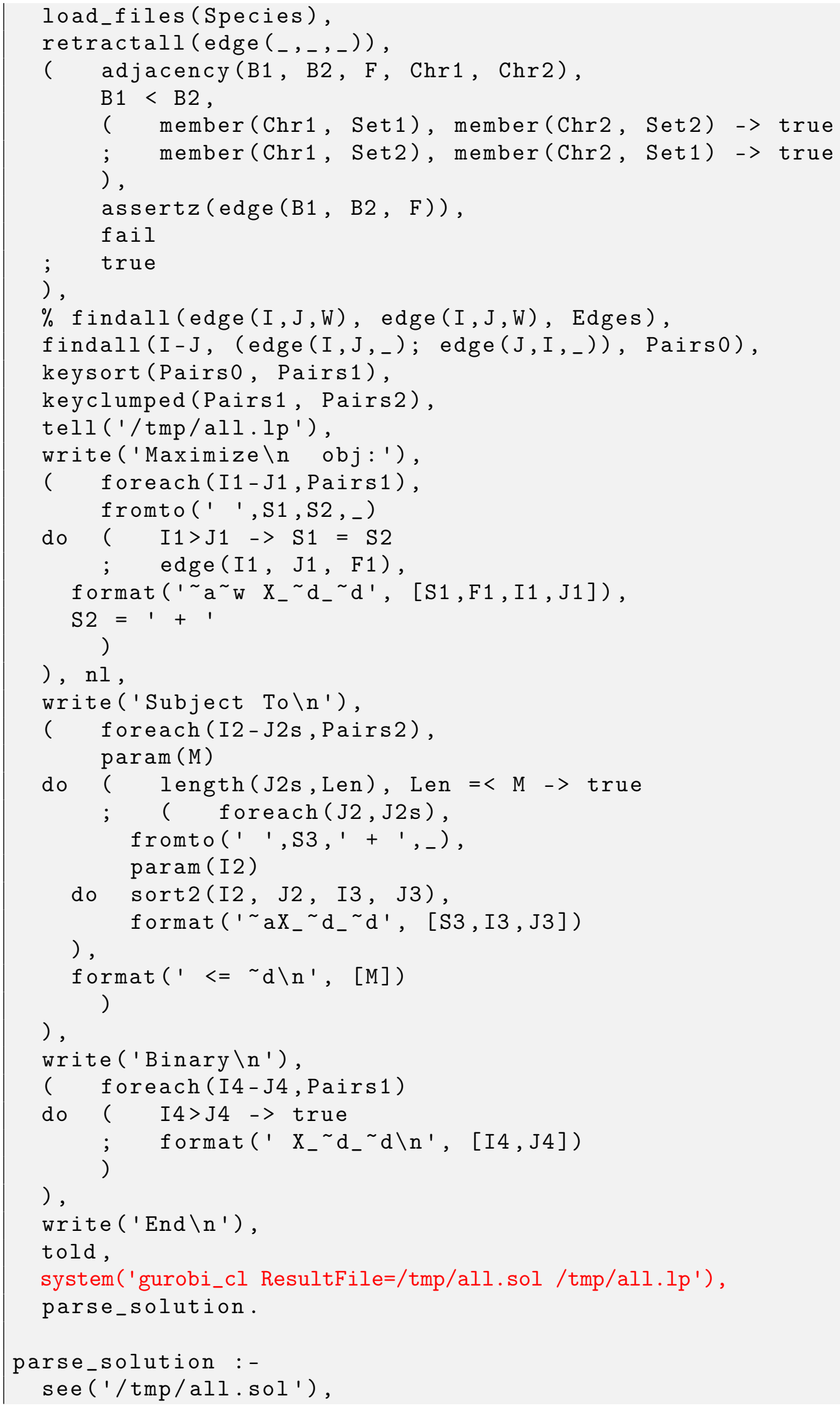




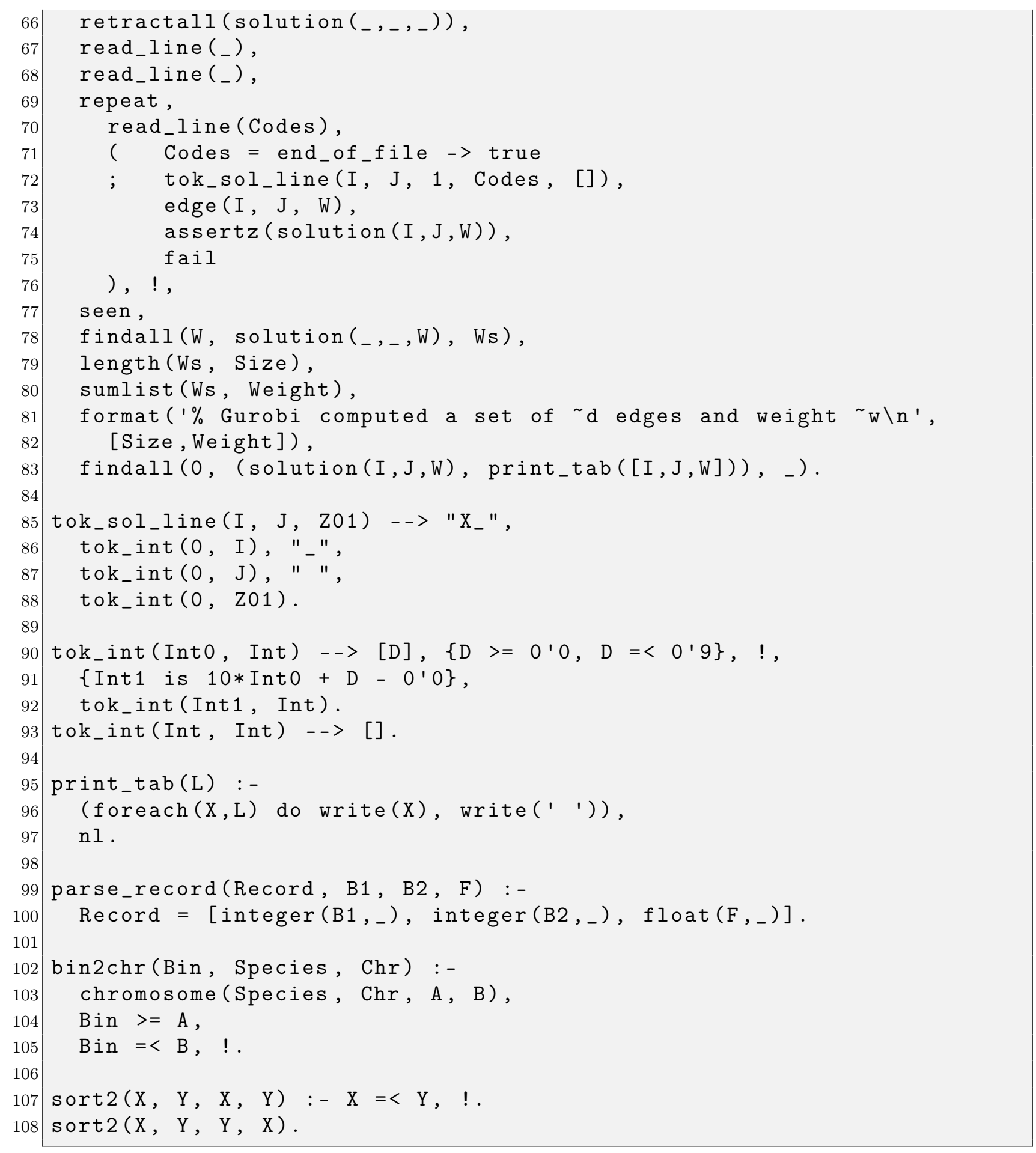

\section{B Example Data Files}


Example Data File 4: An example data file (.dzn file) depicting the integral interaction frequencies from the hypothetical whole-genome contact map depicted in Figure $3 \mathrm{~A}$ utilized by the $\mathbf{C P}$ model.

\begin{tabular}{|c|c|}
\hline $\begin{array}{l}\mathrm{N}=6 \\
\mathrm{map}=\end{array}$ & 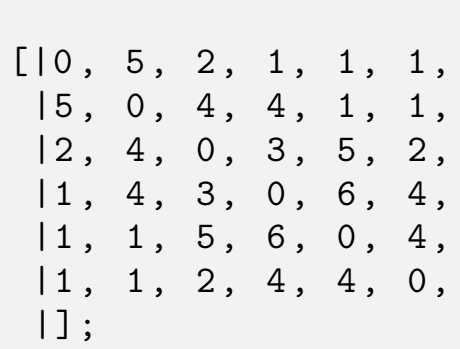 \\
\hline
\end{tabular}

Example Data File 5: An example data file (testMap.csv) depicting the interaction frequencies from the hypothetical whole-genome contact map depicted in Figure 3 A utilized by the GM and IP models.

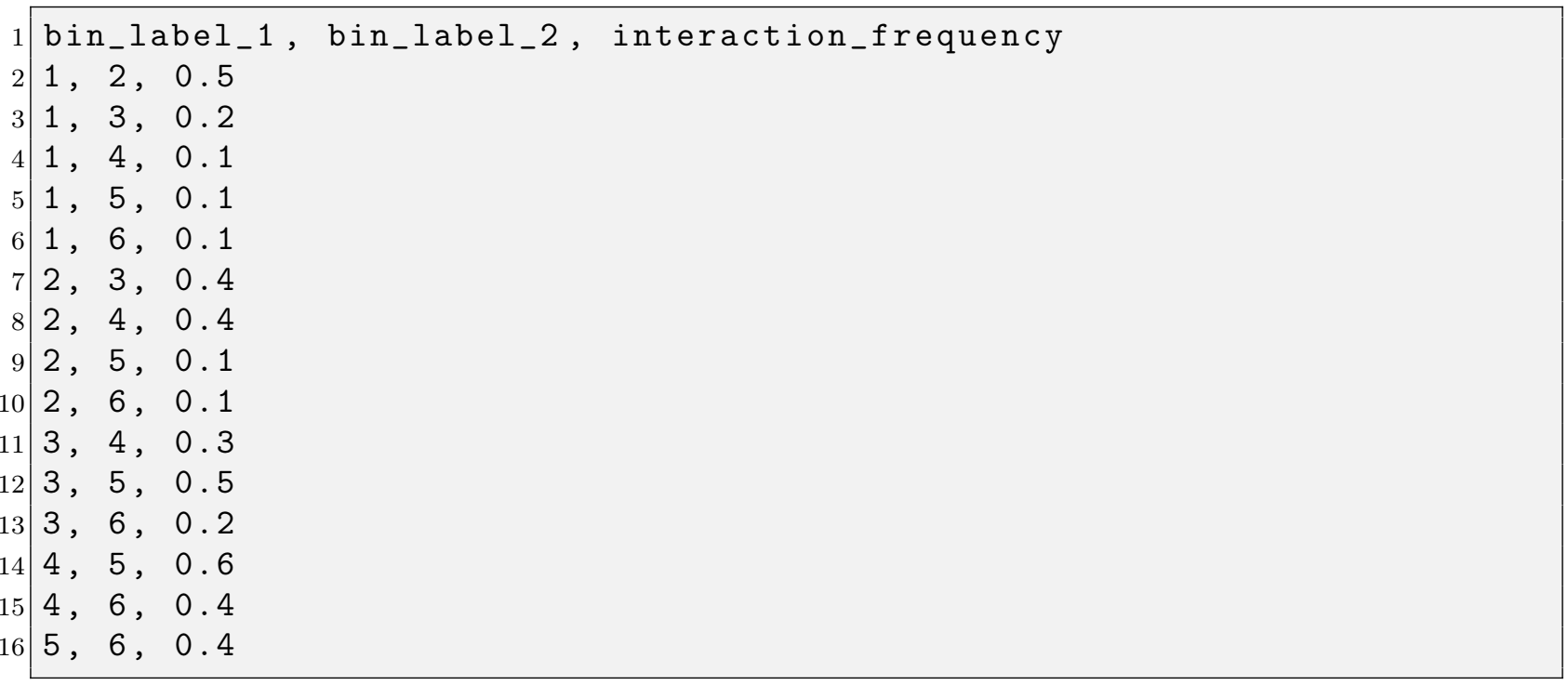

